\title{
Investigation of Effectiveness of Order Review and Release Models in Make to Order Supply Chain
}

\author{
Kaustav Kundu and Alberto Portioli Staudacher \\ Politecnico di Milano, Management, Economics and Industrial Engineering Department, 20133, Milano, Italy
}

\begin{abstract}
Nowadays customisation becomes more common due to vast requirement from the customers for which industries are trying to use make-to-order (MTO) strategy. Due to high variation in the process, workload control models are extensively used for jobshop companies which usually adapt MTO strategy. Some authors tried to implement workload control models, order review and release systems, in non-repetitive manufacturing companies, where there is a dominant flow in production. Those models are better in shop floor but their performances are never been investigated in high variation situations like MTO supply chain. This paper starts with the introduction of particular issues in MTO companies and a general overview of order review and release systems widely used in the industries. Two order review and release systems, the Limited and Balanced models, particularly suitable for flow shop system are applied to MTO supply chain, where the processing times are difficult to estimate due to high variation. Simulation results show that the Balanced model performs much better than the Limited model if the processing times can be estimated preciously.
\end{abstract}

\section{Introduction}

Customization is one of the major challenges faced by most of the high variety-low volume industries [1]. Usually strategies like make-to-order (MTO), engineerto-order (ETO), etc. are incorporated to tackle customization in these companies [2]. In MTO companies, there is lot of variation in the process as well as uncertainty in demand [3]. As a result, the companies sometimes struggle to meet the due dates promised to the customers. In order to tackle uncertainty in demand, many authors suggested Lean techniques like Kanban containers, etc. in MTO companies. Although these Lean techniques are efficient in repetitive companies, they are not proved to be effective in MTO companies where there is high variation in product types as well. Later on some authors tried to adopt Workload control (WLC) techniques like order review and release (ORR) systems particularly for job shop environment. These systems try to absorb the variation in demand and reduces the overall throughput time. As a result, the due dates of the orders are met. These systems are adopted successfully in many jobshop companies. The main focus is on production aspects and on the shop floor activities, particularly how to implement WLC techniques in MTO companies' shop floors. But in MTO companies, orders' value stream consists of the tendering phase, the design phase, the purchasing phase and the production phase and activities are not restricted only in the shop floor. Dealing only in the shop floor activities will ignore the other parts of the whole flow and also important issues like departments' integration and coordination $[4,5,6]$. Therefore, WLC in MTO companies should not be confined only in the shop floor but should also address the other parts of the whole supply chain, from the tendering phase to the assembly stage $[7,8]$, similar to a pure flow shop environment.

There are ORR systems which are specifically designed for jobshop companies. These systems try to hold the orders in the pre-shop pool for some time before releasing them to the shop floor. In this way, the system is able to reduce WIP, any wastages due to cancellation of demand or changes in specifications. There are different kinds of load limiting models developed and implemented in jobshop companies. Few authors tried to apply some of these techniques in pure flow shop environment. Limiting (Upper bound limit) is one such Load limiting model which is proved to work best in the flow shop environment. There are some arguments that these systems should have workload balancing capability which is required for good performance in pure flow shop environment [9]. Therefore, a lean based Balanced release (BLR) ORR system is developed by [7] to control the shop floor process in such kind of environment. This BLR system, capable of balancing workload, shows much better performance as compared to Upper bound limited system in the shop floor of non-repetitive companies. But nowadays as the MTO companies are concerned more about the supply chain, the focus of the paper is on internal supply chain of MTO companies. The pure flow shop can be exactly comparable to the internal supply chain. It will be wise to apply those WLC models in supply chain environment where there is high variation 
in the process. Therefore, the first research question of this paper is: How does the MTO supply chain perform under different ORR systems? To address this question, different simulation scenarios are run for both Limited and BLR ORR systems. Moreover, in MTO supply chain, it is really difficult to predict the expected processing time for each order at each workstation. But the preciseness of processing times can influence the load oriented ORR systems. Therefore, in order to analyze the impact of the preciseness of processing times on the overall performance of ORR systems in MTO supply chain, the second research question is: Does the prediction of processing times precisely influence the performance of ORR techniques in MTO supply chain? To answer this question, the expected processing times are grouped into simple classes [10] and simulation runs are performed for both the models.

The remainder of the paper is organized as follows. The issues in MTO companies and the different ORR systems are described in section 2. The simulation model is described in Section 3. The discussion of the results is then presented in Section 4. Finally, in Section 5, conclusions are drawn along with future recommendations.

\section{MTO companies and ORR systems}

\subsection{MTO companies and their internal supply chain}

The term internal supply chain is used to address the whole set of stages that, from the reception of the customer order to the delivery [11]. In MTO companies' internal supply chains, there are three main classes of business processes: physical, nonphysical and support processes [5]. Physical processes deal with the physical activities on products. Nonphysical processes particularly include the engineering and procurement activities. Support processes are distinguished by being linked to all the nonphysical and physical processes. When a customer orders a product, both physical and nonphysical activities are carried out. All these processes within the supply chain should be controlled using some suitable techniques otherwise the overall performance of the system will be degraded, particularly when there is high variation in the process.

Another key issue that is highlighted in the literature for MTO companies is the due date (DD) setting at the tendering stage [12]. The high variability and incomplete historical data on lead times in MTO companies make the assessment of due dates very difficult [6]. The authors in [13] tried to show the benefits of WLC along with web functionality in supply chain integration. It is proposed in [14] three different procedures for DD quotation depending on the DD type and orders type of the MTO companies: Negotiable DD and fast order, Negotiable DD and slow order, Fixed DD. But DD determination largely depends on the whole flow of the supply chain within the company. Therefore, the main focus should be on the whole flow which handles the output performances. But there is still less attention in the area how to implement suitable techniques to handle high variation in process in MTO companies, particularly in the supply chain [8].

\subsection{Limiting and lean based balanced ORR systems}

Most ORR systems are developed and used mostly in job shops [7], [15]. This system implies avoidance of the immediate release of customer orders, and instead creates a pre-shop pool of orders [16]. The benefits of using ORR systems also include easier control over WIP in a shop floor, better workload balance between the stations, reduces throughput times, increases the reliability of due dates [16]-[18]. ORR systems are in line with lean methodology, as they help to streamline the flow [7]. ORR systems consist of 3 main steps [19]: 1. Order entry phase; 2. Pre-shop pool; 3. Order release phase. Order release phase is most important and there should be an effective selection mechanism at this phase [20]. The existing procedures of order release can be divided into two main categories based on the mechanism of release used [19]: load limited methods; time phased methods. Workload limiting and workload balancing are two order release systems based on load limiting methods These systems have been used the most as they offer an easy implementation and a simple control of the WIP.

The workload norm of order release mechanism limits the load present in the shop floor and the release method sequences and releases the orders based on the parameters decided at the management level in the pre-shop pool. The workload norm helps to achieve an implicit balancing of productive resources. This makes it possible to load up all the machines with a workload as close as possible to their limit [15], [13], [21], [22]. There are three main techniques for the workload control within limited workload order release system: upper bound only [15], [23]; lower bound only [11]; upper and lower bound method [17]. In [24], the authors found out that the upper bound limiting order release system performs the best.

An alternative to the limited workload methods is workload balancing method. This order release method balances the load between the different workstations using extended forecast horizon as well as keeping the overall load inside of certain limits [19]. This method prevents the centers to remain inactive and improves the predictability of the crossing times in the system [15], [25]. The leanbased BLR method developed by [7] and applied to nonrepetitive manufacturing proves to be more effective than limited workload method. According to this method, the focus is on balancing the release workload, disregarding the current disbalance between the working stations. The choice of orders from the pre-shop pool is done by finding the similar amounts of workloads for each workcentre, taking into account the current release period as well as the future ones (extended schedule visibility). A mathematical model is developed and the objective of the mathematical model is to balance the overloading and underloading of loads at each job station over an extended period of time. The details of the mathematical model are available in the article by [7]. 


\section{Simulation model}

\subsection{The structure of the supply chain model}

A supply chain model is constructed from the point of view of a customer request, which follows a route from an order entry phase to the delivery of an order. In this case, a supply chain resembles a flow shop, where orders follow the predefined sequence of departments. The simulation focuses on one of the main specifics of MTO supply chain - uncertainty in prior evaluation of processing times at a high level of variability. The estimation of expected processing times at release can be simplified by grouping the sample of orders into simple classes [10]. The assumptions made are that all the orders are of the same type, so they follow the same route. Python 3.4 Simpy module [26] is used for simulation and CPLEX 12.6 is used to solve the optimization problem for the BLR model.

The ORR systems are tested in the model in order to discover their applicability to MTO supply chain's performance. Before executing the algorithm of ORR system, a current aggregated workload present in a MTO supply chain and at each department is measured. The workload includes 2 main components: a direct workload of a department, which is found in the queues of each department; and indirect workload, which is the sum of processing times of all the orders in the system that sooner or later have to pass through this department (often referred as an upstream load). Two different ORR systems, Upper bound Limited Workload and BLR Workload, as described in [7] are used for order release in this paper. For simulation, the basic assumptions and the parameters for both the models are almost the same as that given in the article by [7] with little bit modification. According to the classifications by [19], the characteristics of both the ORR systems applied to the SSC simulation model are the same except for two factors. Aggregation of workload for Limited Workload is load at each department whereas for BLR Workload is total load in the supply chain; Schedule visibility for Limited Workload is limited whereas for BLR Workload is extended.

\subsection{Input parameters}

In order to simplify the model, the randomness in orders is ignored and they are assumed to be constant. Based on previous studies, the arrival of orders follow a Poisson distribution on average every 16 minutes. Thus on average there are 30 orders per day and all 30 orders arrive to the system just at the beginning of each release period (day in this case). It is decided to use a lognormal distribution of processing times, which is quite common in MTO supply chain [27]. The processing times at each department are assumed to be 29.7 minutes long on an average. As the objective of this paper is related to high variation situation, coefficient of variation of 0.8 is assumed here. Considering the assumptions, the input data is generated with the use of Minitab 17 software. The generated values represent the actual processing times, occurred at each stage of a supply chain. But they cannot be used for planning purposes, as they are not known in advance, before the request is processed. Thus the expected processing times, which are estimated when the request arrives, should be also considered. To do so, each sample is divided into equal parts - classes, and the mean of each class is found. Each class' mean is used for planning purposes as an expected processing time, which is estimated on the requests' arrival time. To answer the second research question, different number of classes are tested. Thus, for a low precision level there are 3 classes of expected processing times: short, medium and long. And for a high precision level in estimating the expected processing times, 7 classes are considered.

\subsection{Conduction of experiments}

As there is huge variation in processing times of different orders, the system workload cannot be calculated in terms of number of orders. Instead it is assumed to be the total capacity of the entire supply chain and it is defined in terms of minutes. To determine the optimal system workload, some preliminary simulation runs are performed. Simulation is started with very high system workload and then slowly the workload is decreased to lower values. Around 18000 minutes, the performance measures of the system are found to be optimal. Therefore, 18000 minutes is considered to be the system workload for the rest of the experiments. Warm-up period corresponds to the time interval, after which the system becomes stable. The most used method for the calculation of the warm-up period length is the graphical method described by [28]. To determine a warm-up period and length of the simulation runs, an experiment is conducted at 18000 minutes system workload. During the first 300 days the MTO supply chain performance is not stabilized. As the simulation is of a steady-state type and its initial state doesn't represent the real behavior of the system, a warm-up period is set to 300 days, until the output stabilizes. In order to avoid any possible errors, the length of the run is set to 500 days. These values are used for all the experiments. The performances [22] that are measured are: Average Gross throughput time (GTT) total time from arrival of order till its delivery including waiting time in the preshop pool; Average Supply chain time (SCT) - total time from release of order for actual processing in the supply chain till its delivery; Average Output workload - the load of orders that are released per period.

The experiments are planned in accordance to the research questions of the paper. First set of experiments are conducted with the actual processing times for both the models. The second set of experiments are conducted in order to simulate the MTO supply chain with different precision levels in processing times estimation. With the obtained results from the first set of simulation runs, it will be possible to compare the performance of both models in MTO supply chain. With the obtained results from the second set of experiments, it will possible to analyze the impact of uncertainty in processing times on the performance of upper-bound Limited ORR system 
and BLR ORR system. In MTO supply chain, the workload does not remain stable. Therefore, it will be wise to test the models under all kinds of situation. Different target workload norms (defined in minutes), which range from very short queues in a MTO supply chain to very long ones (960 to 3500 minutes) are defined to limit the load for each job station in the supply chain. The actual output performances are obtained in minutes but for better understanding, they are converted into hours.

\section{Results and discussion}

The results of the simulation runs that are carried out to answer the research questions are shown in this section. Figure 1 shows the variation of GTT with respect to SCT. As the workload norms increase, less orders remain in the pre-shop pool and more orders flow into the actual system, thereby decreasing the throughput time of the orders upto certain extent. Therefore, GTT first decreases with SCT and then becomes more or less stable. It can be also seen from the figure that at same SCT, the GTT for BLR model is much lower than that of the Limited model. The difference in performance increases as the workload norms become tighter, i.e., situation when there is short queue within the supply chain. It convinces that the BLR model is better than the Limited model, especially at tighter workload norms. Therefore, it answers our first research question.

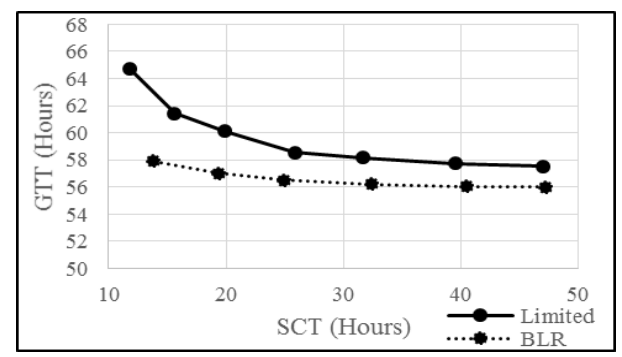

Figure 1. Variability index (sigma/mu) of $80 \%$ for actual processing time.

Figure 2 shows that the difference in performance between the Limited and BLR models at low precision level (Class 3). The GTT obtained from both the models at different workload norms are much higher than that obtained using actual processing time information. At some SCT, there is little bit difference between the models but the overall difference in performance is not so significant at low precision level. Therefore, the BLR model does not perform well when it is difficult to predict the processing times precisely.

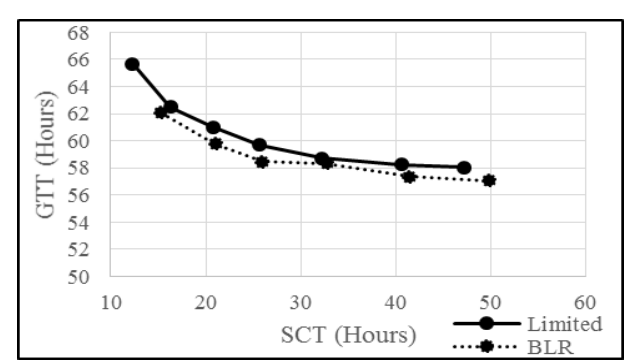

Figure 2. Variability index (sigma/mu) of $80 \%$ for low precision (Class 3 ) processing time.
Figure 3 shows the difference in performance between the two models at high precision level (Class 7). The performance of Limited model is similar to that obtained with actual processing times. But for BLR model, the performance is little bit different at tighter workload norms with that at the actual processing times. The trend of the curves are more or less similar to that in Figures 1 and 2. Both the models perform much better at high precision level than at low precision level. Moreover, there is not significant differences in throughput times for both the models with actual processing times as well as with high precision level. From Figures 2 and 3, it is revealed that BLR model performs better than Limited model when processing times can be estimated accurately at the planning stage. This answers our second research question.

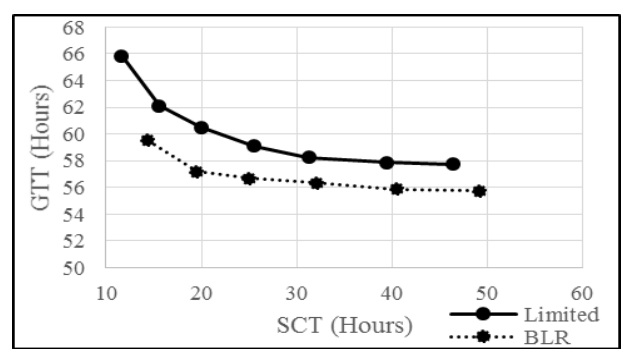

Figure 3. Variability index (sigma/mu) of $80 \%$ for high precision (Class 7) processing time.

Order release decision solely depends on the processing times [29]. Both BLR and Limited models depend on the total load, in terms of processing times. Thus any wrong prediction in processing times of different orders will degrade the performance of BLR model. Therefore, BLR model performs better than Limited model only when the processing times can be estimated correctly at the planning stage.

\section{Conclusion}

This paper looks at the processes of the MTO companies from the point of the internal supply chain as a whole. This gives an opportunity to look at the flow along these processes and eliminate waste, thus leaning the supply chain, and not only its separate stages. Due to variation in processing times and uncertainty in demand, it is really difficult to manage the internal supply chain which directly impacts the customer requirements. In jobshop environment, similar kind of situation is tackled by using workload ORR techniques. But few authors [7] stated the importance of workload ORR techniques in a pure flow shop environment which is suitable for high customization. MTO supply chain is similar to a pure flow shop environment. Continuing the studies of [7] on ORR systems, which proved to be effective in leaning the processes in non-repetitive manufacturing, two types of ORR systems are applied to the MTO supply chain. The Limited and BLR models are run at different workload norms in order to capture all possible situations at 18000 minutes system workload. It is seen form the simulation runs that GTT decreases with increase in SCT, which is quite logical. At actual planning situation, the BLR model performs much better than the Limited model, 
particularly at tighter workload norms. But in reality it is very difficult to predict the processing times of different orders beforehand. Therefore, processing times are estimated at low and high precision levels and both the models are run at different workload norms. It is seen that the BLR model performs better than the Limited model only when the processing times can be estimated more closely to the actual data. As both BLR and Limited models depend on the load (processing times in this case), the accuracy of the load plays an important role in the performance of ORR systems in MTO supply chains. Therefore, the important contribution of this paper is that it is quite reasonable for the MTO companies to use the workload ORR systems to improve the performance of their internal supply chain but there should be some way to predict the data as close as possible to the original situation in order to obtain the maximum benefits.

This paper is purely theoretical and is based on statistical distributions and generalized model. Though the results are statistically significant, the processing times in the companies might have different distributions and thus have different effects on the performance. But the findings of this paper create a strong base for further research on the applicability of ORR systems to different aspects of MTO supply chain. Here the study is concerned only with the pure flow along the internal supply chain with simple configuration but future studies can concentrate on other factors like the parallel processing of orders, multiple resources, transportation, etc. It will be quite interesting to see how the simulation model could be adapted to the real case. Therefore, another suggestion for further research is to collect data from a company or companies and confirm these theoretical findings empirically.

\section{References}

1. P. R. Dean, Y. L. Tu, and D. Xue, Int. J. Prod. Res., 47, 4, pp. 1071-1087 (2009)

2. E. R. Melchert, M. A .de Mesquita, and P. G. Francischini, 12th Int. Conf. Indus. Eng. Opers. Man. (ICIEOM), Fortaleza, CE, Brasile (2006)

3. W. J. Hopp, and M. L. Spearman, Manufac. Serv. Opers. Man., 6, 2, pp. 133-148 (2004)

4. B. G. Kingsman, and A. Mercer, Inter. Trans. Oper. Res., 4, 1, pp. 251-257 (1997)

5. C. Hicks, C. F. Earl, and T. McGovern, IEEE Trans. Eng. Man., 47, 4, pp. 414-423 (2000)

6. C. Hicks, T. McGovern, and C. F. Earl, Inter. Int. J. Prod. Econ., 65, pp. 179-190 (2000)

7. A. Portioli, and M. Tantardini, Int. J. Prod. Res., 50, 12, pp. 3257-3273 (2012)
8. M. Thurer, M. Stevenson, C. Silva, M. J. Land, L. D. Fredendall, and S. A. Melnyk, Prod. Oper. Man., 23, 3, pp. 463-476 (2014)

9. R. Germs, and J. Riezebos, Int. J. Prod. Res., 48, 8, pp. 2345-2360 (2010)

10. M. Thurer, M. J. Land, and M. Stevenson, Inter. Int. J. Prod. Econ., 147, pp. 180-188 (2014)

11. S. Chopra, and P. Meindl, "Supply Chain Management: Strategy, Planning and Operations", second ed. Prentice Hall, Upper Saddle River, NJ (2007)

12. D. Corti, A. Pozzetti, and M. Zorzini, Inter. Int. J. Prod. Econ., 104, pp. 536-554 (2006)

13. M. Stevenson, and L. C. Hendry, Inter. Int. J. Prod. Econ., 104, 2, pp. 676-693 (2006)

14. M. Zorzini, D. Corti, and A. Pozzetti, Inter. Int. J. Prod. Econ., 112, pp. 919-933 (2008)

15. M. J. Land, and G. J. C. Gaalman, Inter. Int. J. Prod. Econ., 56-57, pp. 347-364 (1998)

16. S. A. Melnyk, and G. L. Ragatz, Int. J. Prod. Res., 27, 7, pp. 1081-1096 (1989)

17. L. Hendry, and B. Kingsman, Int. J. Oper. Prod. Man., 11, 6, pp. 6-16 (1991)

18. D. Bergamaschi, R. Cigolini, M. Perona, and A. Portioli, Int. J. Prod. Res., 35, 2, pp. 399-420 (1997).

19. I. P. Tatsiopoulos, Comp. Indus., 1997, 33, pp. $335-$ 344 (1997)

20. I. Sabuncuoglu, and H. Y. Karapinar, Inter. Int. J. Prod. Econ., 62, 3, pp. 259-279 (1999)

21. P. Henrich, M. Land, and G. Gaalman, Inter. Int. J. Prod. Econ., 90, pp. 187-198 (2004)

22. M. Land, Inter. Int. J. Prod. Econ., 40, pp. 196-209 (2006)

23. W. Bechte, Int. J. Prod. Res., 26, 3, pp.375-395 (1988)

24. R. Cigolini, and A. Portioli-Staudacher, Prod. Plan. Contr., 13, 7, pp. 602-613 (2002)

25. H. P. G. Van Ooijen, Inter. Int. J. Prod. Econ., 56-57, pp. 661-675 (1998)

26. Land, M. J., M. Stevenson, M. Thurer, and G. J. C. Gaalman, Inter. Int. J. Prod. Econ., 168, pp. 257-266 (2015)

27. T. M. Choi, and T. C. E. Cheng, "Supply Chain Coordination under Uncertainty", Springer Science \& Business Media, 14 Aug (2011)

28. P. D. Welch, "The statistical analysis of simulation results", in The Computer Performance Modeling Handbook, ed. S. Lavenberg, pp. 268-328. New York: Academic Press (1983)

29. M. Thürer, and M. Stevenson, Int. J. Prod. Res., DOI: 10.1080/00207543.2016.1177672 (2016) 